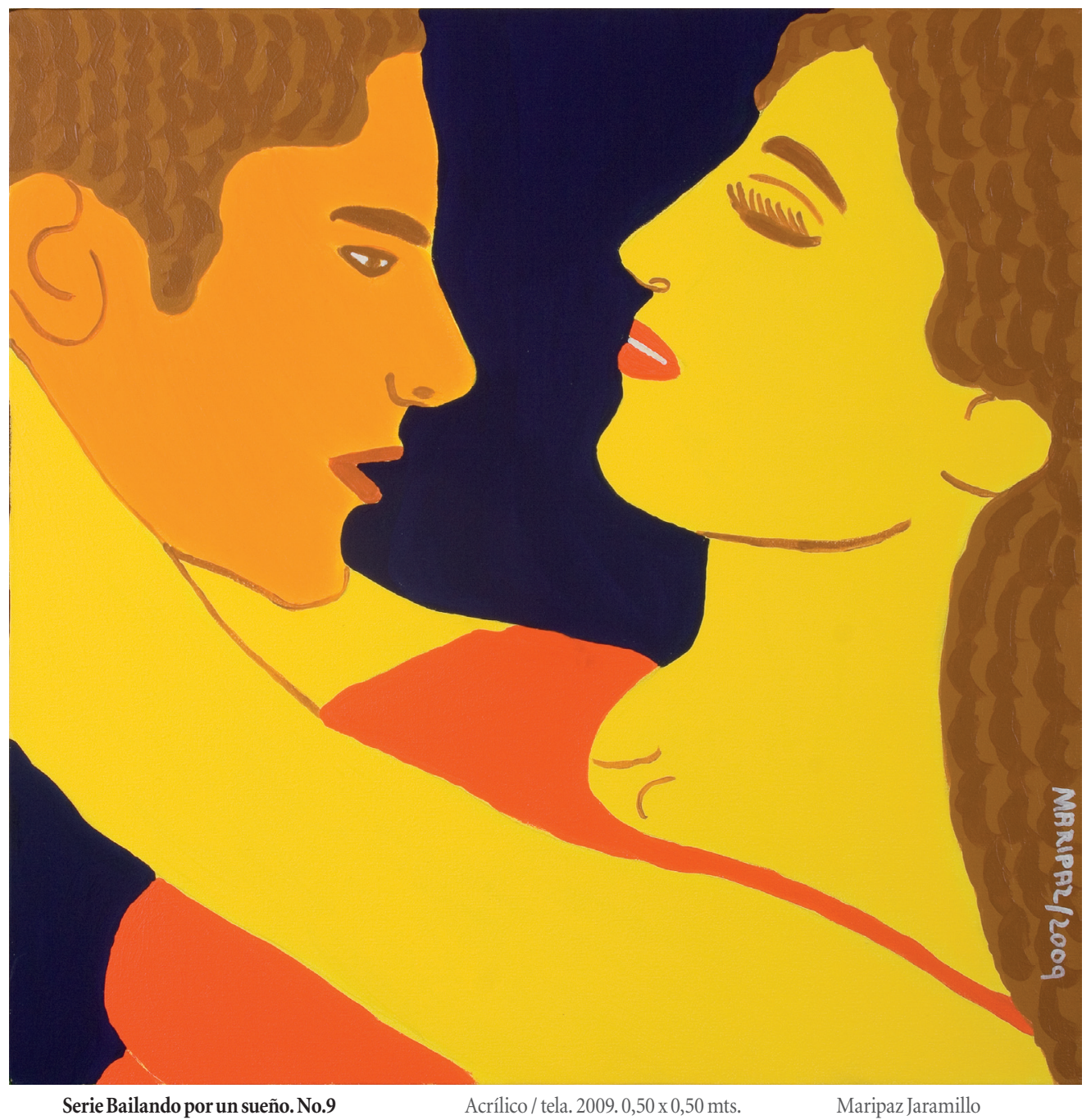




\title{
Revisitando la crítica a la teoría fundamentada (grounded theory)
}

\author{
Fecha de recepción: 18 de enero de 2011 - Aprobación: 15 de marzo de 2011
}

\section{Eduardo Escalante Gómez}

\begin{abstract}
Resumen
El propósito de este artículo es examinar algunas de las críticas relacionadas con la perspectiva de investigación desde la teoría fundamentada. Al interior del enfoque interpretativista se encuentran numerosos métodos para construir conocimiento y cada uno tiene su fundamento filosófico, prácticas, y métodos de interpretación. La teoría fundamentada es una de tales metodologías. Sin embargo, ésta en particular, a veces se percibe como cercana al positivismo y al funcionalismo, que enfatiza los procedimientos metodológicos para analizar datos y generar teorías.

Este artículo discute la teoría fundamentada, principalmente desde sus presupuestos filosóficos de la metodología y procede a examinar algunos de los conceptos claves asociados con este enfoque.
\end{abstract}

\section{Palabras claves}

Método, epistemología, teoría, investigación cualitativa.

\begin{abstract}
The purpose of this paper is to examine some criticisms that are related to the research perspective from the Grounded Theory. Within the interpretivist approach, there are numerous methods for building knowledge and each one has its own underlying philosophies, practices, and methods of interpretation. The Grounded Theory is one of those methods. However, this one in particular, is sometimes perceived as being close to positivism and functionalism, which emphasizes methodological procedures in order to analyze data and create theory.

This article discusses the grounded theory, mainly from its philosophical assumptions of the methodology and examines some of the key concepts related to this approach.
\end{abstract}

\section{Keywords}

Method, epistemology, theory, qualitative research.

\section{El análisis cualitativo a partir de la Teoría Fundamentada (Grounded Theorn)}

La teoría fundamentada ha tenido un enorme desarrollo en el procesamiento de datos cualitativos y la generación de teoría a partir del libro The Discovery of Grounded
Theory de Barney Glaser y Anselm Strauss en 1967. A pesar de las innumerables críticas a esta teoría, continúa siendo usada en muchas investigaciones, tesis doctorales, y hay un sinnúmero de artículos publicados en importantes revistas internacionales en 
diferentes idiomas. Se podría decir que es uno de los métodos más influyentes en investigación cualitativa en las últimas décadas, aplicado en su singularidad o combinado con otros métodos como los fenomenológicos. Según Miller y Fredericks (1999) ha llegado a ser el "paradigma de elección" para los investigadores cualitativos.

Este tipo de método se ha hecho popular porque satisface una necesidad. En discusiones con tesistas e investigadores, a menudo se escuchan argumentos del tipo: "con la teoría fundamentada puedo procesar la información de mis entrevistas"; "puedo trabajar con el significado"; "puedo ordenar y categorizar la información”; y otros más. En general, no se escuchan argumentos metodológicos más profundos y precisos, al parecer, todo resulta ser una cuestión de pragmatismo intelectual.

Brevemente, el propósito de la teoría fundamentada es generar teoría usando un enfoque inductivo. Traslada su eje de preocupación desde la contrastación de teoría a la generación de teoría. El investigador procede sin una hipótesis. Los datos brutos son estudiados y codificados por el investigador mediante un determinado procedimiento analítico que se inicia con su codificación. A partir de los códigos, el investigador hace emerger las categorías. El investigador puede fracturar los datos de diferentes maneras

\section{Reseña de autor \\ Eduardo Escalante Gómez (Chile) \\ Universidad del Aconcagua (Argentina). \\ escalante.gomez@gmail.com}

Magíster en Ciencias Sociales (Universidad de Gales, Gran Bretaña), docente de las maestrías de Criminología y Terapia Sistemática, investigador principal y director Instituto de Investigaciones, Facultad de Psicología, Universidad del Aconcagua (Argentina). Autor de diversos artículos científicos indexados en SciELO y Redalyc. con el fin de lograr una mayor comprensión de estas categorías, sus propiedades y sus interrelaciones. Cada categoría tiene propiedades; sus descripciones se agregan en el estudio y análisis de los datos hasta alcanzar un punto en el que los datos no aportan más información. Se dice entonces que la categoría está saturada. Este proceso permite que emerjan las relaciones entre las categorías, las cuales pueden ser identificadas y se puede concluir que una categoría que organiza los datos tratándose de una categoría nuclear, denominada por algunos como metacategoría. Realizado este proceso, el investigador inicia lo que se denomina un proceso de sensibilización teórica, que consiste en generar la teoría a partir de los datos. Se realiza un proceso detallado de verificación de los datos mediante lo que se denomina método de la comparación constante. Esto permite al investigador verificar las hipótesis emergentes que conducirán al desarrollo de la teoría sustantiva. Esta puede ser relevante más allá del campo de estudio, en este caso un proceso mayor de abstracción pude conducir a la formación de la teoría formal. La teoría generada debe ser relevante al área y/o problema en cuestión, debe calzar con los datos, y debe funcionar, es decir, producir explicación, comprensión, y ser predictiva. Esto no prueba que la teoría sea correcta, sino más bien que la teoría es un conjunto de hipótesis integradas. No se trata de una palabra final, pues son posibles nuevos desarrollos. En todo momento, se destaca el proceso inductivo y el procedimiento analítico a realizar.

\section{Teoría Fundamentada y Epistemología}

Dependiendo de la posición del investigador sobre la verdad y la realidad, hay varios enfoques metodológicos que se pueden 
adoptar (Cohen y Manion 1994). Además, la forma de la teoría fundamentada que se adopte depende de una clarificación de la naturaleza de la relación investigador - participante y de la explicación de lo que podemos conocer (Mills et al. 2006).

Para asegurar un diseño investigativo consistente, este debe ser congruente con las creencias del investigador sobre la naturaleza de la realidad. La teoría fundamentada se ha desarrollado de algún modo en direcciones divergentes (Guba y Lincoln 1994): el modo clásico de Glaser se caracteriza como crítico realista y objetividad modificad" (Annells 1997a 1997b), y el de Strauss y Corbin (1991) como una posición relativista y subjetivista. Charmaz (2000) sugiere que ambos adhieren a una ontología realista y a una epistemología positivista pero con algunas diferencias.

La epistemología positivista se basa en la suposición que el mundo social se puede investigar de manera similar a como se investiga el mundo natural. Glaser asume una realidad objetiva y externa. Es un observador neutral de los datos bajo una manera objetiva y por consiguiente descubre la teoría (Glaser 1992, Glaser1999, Glaser 2001). La realidad es entonces libre del contexto y generalizable (Lincoln y Guba 1985), La visión de Strauss y Corbin, que ha sido denominada teoría fundamentada reformulada, asume una realidad externa objetiva y busca una recolección de datos desprejuiciada. Ellos dan voz a los que responden, reconociendo su interpretación de la realidad (Charmaz 2000).

Así mismo, el paradigma post positivista argumenta que aunque la realidad existe para ser descubierta por la indagación, no es nunca aprehensible de manera perfecta
(Guba y Lincoln 1985). Según Charmaz, es posible usar la teoría fundamentada sin adoptar las posturas anteriores y agrega una nueva: la teoría fundamentada constructivista que estudia a las personas en sus ambientes naturales. No podemos conocer nunca la realidad, solamente podemos tener interpretaciones de ella y por tanto nuestras ideas determinan lo que conocemos. Un enfoque constructivista ve los datos y sus análisis como creados a partir de experiencias compartidas y relaciones con los participantes y otras fuentes de datos. Se funda en una ontología que señala que lo que existe depende de lo que los individuos perciben que existe. Se rechaza la existencia de una realidad objetiva, por consiguiente existen tantas construcciones interpretativas como existan individuos aunque muchas serán compartidas (Guba y Lincoln 1985). Las realidades sociales no son separables del investigador, esto porque los investigadores construyen los mundos que investigan.

El procedimiento de la teoría fundamentada variará enormemente según la postura epistemológica que se sustente, tanto con relación a la recolección de los datos como con los procesos interpretativos de los resultados. Por ejemplo, desde una postura realista, el investigado no constituirá una parte importante del proceso, en cambio sí lo será desde el punto de vista constructivista. Es innegable el valor de un esquema elemental de descripción, ordenamiento de datos, una codificación, y de algún modo, el de categorización, pero es cuestionable, por ejemplo, el proceso de generación de teoría según la interpreta la teoría fundamentada. El uso de herramientas analíticas claramente posiciona al investigador como autor, quien 
Para asegurar un diseño investigativo consistente, este debe ser congruente con las creencias del investigador sobre la naturaleza de la realidad. reconstruye el significado en el proceso investigativo y el proceso es opaco hasta que el investigador asigne dicho significado y su especificidad (Corbin \& Strauss 1990).

\section{Revisiones de la teoría fundamentada}

La revisión y adaptación de la teoría fundamentada es un tema recurrente en los investigadores cualitativos. Entre los autores que han planteado una revisión y desarrollo de la teoría fundamentada son Dey (1999), que, independientemente de sus críticas, ve a esta teoría como la capacidad de desarrollar y ofrecer un camino intermedio entre la teorización ideográfica y nomotética; Layder opta por una modificación, proponiendo finalmente un enfoque realista o una teoría adaptativa (Layder, 1993); Haig (1995) que también busca una adaptación; Charmaz (2000) quien toma un camino intermedio entre postmodernismo y positivismo, representa una contribución importante desde una postura constructivista. Clarke (2005) que argumenta a favor de un enfoque de análisis de datos que refleje una preocupación por cómo se producen los discursos y cómo nos constituimos a través de ellos. Este autor trata de encontrar puntos de articulación entre Strauss y Foucault y Plantea un método de análisis situacional que permita ubicar a los participantes en la investigación en su mundo social: un mundo que está lleno de otros actores.

Otro camino de adaptación de la teoría fundamentada se propone a partir del uso de metodologías combinadas. A modo de ejemplo, Annells (2006) propone una triangulación entre dos enfoques cualitativos: fenomenología hermenéutica y teoría fundamentada. La autora en su estudio argumenta que no solamente un fenó- meno se interpreta según los posibles procesos sociales que ocurren y se relacionan con dicho fenómeno, sino también a través de la comprensión sobre los posibles significados de la experiencia existencial del ser humano sobre este. La autora hace una interesante adaptación de la comparación de una versión constructivista de la teoría fundamentada y la fenomenología hermenéutica con influencia de Gadamer.

\section{Limitaciones de la teoría fundamentada}

Sin embargo, no se ve claramente que sea posible una vía intermedia o nuevas versiones. Hay tres conceptos que permanecen cuestionables: teoría, fundamentada (grounded) y descubrimiento.

\subsection{Concepto de teoría}

Respecto del concepto teoría se puede señalar que no ha sido un foco de crítica a la teoría fundamentada. No obstante, el hecho que se denomine teoría fundamentada (grounded theory) hace que surjan algunas preguntas sobre qué es una teoría, qué se espera de una teoría y el por qué los investigadores esperan que sus métodos usados para construir sentido, se llamen teoría.

Se ha considerado la teoría fundamentada como una teoría general del Método Científico preocupado de la generación, elaboración, y validación de la teoría de la ciencia social. Se argumenta que para esto debería reunir cánones en aras de hacer buena ciencia como lo son consistencia, reproducibilidad, generabilicidad, entre otros, aunque estas nociones metodológicas no deberían ser entendidas en un sentido positivista. Se trataría de comprender un fenómeno y derivar la teoría inductiva- 
mente desde los datos, sometiéndola a elaboraciones teóricas y juzgarla adecuada a su dominio según un determinado número de criterios evaluativos.

La postura de Glaser y Strauss (1967) indicaría que la investigación cualitativa es válida, apropiada y necesaria; el desarrollo de la teoría se ve como sine qua non en la indagación seria, para lo que se requiere de una metodología que permita demostrar cómo se desarrolla la teoría. La preocupación por la teoría se vincula principalmente con la noción de inducción: la teoría es parte de un aparato canónico que permite hacer inferencias y elaborar explicaciones basados en procesos inductivos. El concepto de inducción requiere varias precisiones, como señala González Echeverría (2005, pág.25). Esta antropóloga, que tiene una visión positiva de la teoría fundamentada, señala que "el problema está en que, al autodefinirse como inductivistas, hacen pensar a algunos de sus lectores, obviamente no a todos, que la inductiva como investigación emergente extraería las hipótesis de los datos". Y agrega que, "al huir del término hipótesis, porque está ligado a la gran tradición, pierden parte de lo que se había aprendido sobre la forma de manipular estos constructos, y no sólo es difícil poner a prueba hipótesis con datos de campo previos a su formulación, sino que para buscar verificaciones y refutaciones hay que seguir caminos distintos".

Por otro lado, se puede complementar que ya ha quedado atrás la secuencia inductivista que pensaba en el conocimiento construido en tres etapas: toma de datos, comparación, y teorización. Aunque es legítimo mencionar que en la formulación de Glaser y Strauss no hay una separación absoluta entre el proceso de recogida de datos y el de análisis, por lo cual hay contrastación continua (González Echeverría, 2006).

La palabra teoría ha adquirido diferentes significados: explicación, reflexión personal, principio orientador, supuestos epistemológicos, desarrollo de un argumento, entre muchos otros (Thomas 1997). Siguiendo a Miller y Fredericks (1999, p. 539), se puede comentar que la teoría fundamentada constituiría un enfoque de lógica del descubrimiento con características predictivas y una variante a la inferencia de la mejor explicación. En cuanto a la conceptualización de predicción y explicación se adhiere, de algún modo a las expectativas positivistas y funcionalistas, aunque se trata de ir más allá de Merton y Parsons.

Se mezcla inspiración con predicción y no le es suficiente la comprensión de los fenómenos. Se intenta generar teorías de alcance medio, más allá de la gran teoría. No obstante, valga comentar que el investigador debería ser cuidadoso, por el hecho de que, al crear algo denominado teoría, junto con un conjunto de procedimientos necesarios para hallarla, podría inhibir más que liberar el descubrimiento, según lo pretende la teoría fundamentada, esto es, habría que evitar que en función del orden se sacrifique la fertilidad.

En el contexto de análisis de Charmaz (1995, p. 28), se reconocería una distinción entre teoría débil y teoría fuerte, dado que para ella, la teoría fundamentada, sería una teoría de rango intermedio que explica la conducta y los procesos. Resulta problemático el concepto de explicación y el de rango intermedio dado que las teorías del conocimiento local no explican nada porque no hay procedimiento para hacerlo; sí ayudan 
a entender, no se trata de una ambición menor, pero la teoría fundamentada busca concretar su gran ambición: explicar. Para que la teoría tenga valor, debería involucrar algo más que la identificación de los patrones cotidianos y de los ejercicios heurísticos tácitos dado que tales patrones y ejercicios son humanos por excelencia todo el tiempo. El investigador debería evitar la unión de subjetivismo con cualquier otra parte, teniendo cuidado con igualar descripción con análisis, análisis con explicación, explicación con descripción y teoría con todo eso.

El tema de la teoría no es fácil resolverlo dado que en Ciencias Sociales a lo menos se distingue la posición de aquellos que piensan que la teoría está formada por herramientas para pensar (Nadel, 1957) es decir, sirven para mapear un área problemática; $y$ la posición de quienes piensan que la teoría es un conjunto de afirmaciones que nos dicen algo nuevo sobre el mundo social y que puede ser aprobado o desaprobado por la investigación empírica. Una distinción similar se puede encontrar en Bourdieu quien afirma que él nunca busca "hacer" o "construir" una teoría, pero que no hay dudas de que en su trabajo hay teoría o, mejor dicho, herramientas de pensamiento visible a través de los resultados alcanzados (Bourdieu, in Wacquant, 1989 p. 50).

Según Glaser y Strauss (1967), la adecuación de una teoría no puede estar divorciada del proceso de investigación en el que se genera, dado que muchas hipótesis y conceptos no sólo provienen de los datos, sino que se obtienen con relación a los datos en el curso de la investigación. En otras palabras, se trata de una teoría que se genera en el proceso de investigación, no de un sistema hipotético- deductivo donde se trabaja a partir de unos presupuestos a priori de los que se deducen lógicamente las hipótesis de trabajo.

El problema con la teoría fundamentada es el vínculo de regularidad y generalización con inducción. Se mezclan las expectativas de una clase de teoría con otra. Se argumenta en términos de un conjunto de procesos naturales de comprensión e identificación de patrones con la explicación típica de las ciencias naturales. Glaser y Strauss son explícitos en declarar que la teoría fundamentada busca predicciones relevantes, explicaciones, interpretaciones y aplicaciones. Según estos autores, mediante el uso de la teoría fundamentada se logra que la inducción permita derivar teoría, la que permitiría ordenar y predecir.

Lo que se observa es que las interpretaciones ofrecen solamente una narrativa y nada más, lo que no significa que esté mal si lo miramos, por ejemplo, desde la perspectiva de la hermenéutica de Gadamer. Lo que resulta cuestionable es suponer que el investigador provisto de un conjunto de procedimientos y técnicas (saturación, muestreo teórico) transformará una comprensión de las narrativas o sucesos en una especie de teoría inductiva. Resulta problemático, por decir lo menos, suponer que la objetividad procedimental nos conduciría a la objetividad ontológica, que no es otra cosa que ver las cosas de la manera como realmente son.

Cuando se sostiene la validez de la indagación cualitativa, se está asumiendo la validez de la interpretación y la comprensión en el mundo social. Esta comprensión se construye a partir de lo que nosotros como personas, interpretamos lo de otros: sus palabras, sus acciones. Las interpretaciones se construyen a partir de lo que significa ser 
humano. Un método no sustituirá los esenciales de esta humanidad. La interpretación no busca, produce sentido.

Se podría decir que, de algún modo, la teoría fundamentada es un enfoque no cualitativo de indagación cualitativa. Su esfuerzo pareciera el asegurar un nicho epistémico seguro, para lo cual se construye un conjunto de procedimientos, es una necesidad más de conmensuración que de conversación; más de medición que de memoria, por preferencia por la estructura más que por el suceso.

En síntesis, el problema no es con las metas de la indagación cualitativa postulada por la teoría fundamentada, sino con la búsqueda de una teoría particular, por la noción de teoría, por el significado de la teoría y por lo que esperamos de ella. Pero también con la idea de poder producir teoría a partir de los datos, como lo hacen Strauss y Corbin. Los hechos no hablan a menos que los interrogue una teoría; los significados no proliferan a menos que actúe un método interpretante (expresable, comunicable y replicable en tanto método). Si se logra articular un problema de modo que tenga una estructura de propiedades conocida, en muchos casos será posible determinar a priori qué clase de soluciones admite o si no admite ninguna. Este tipo de consideraciones no se establecen en la teoría fundamentada.

Creemos que es una noción ilusoria el que la teoría pueda provenir exclusivamente de los datos mismos, como si éstos hablaran, o como si los hechos contuvieran en sí las claves que permiten su interpretación. Sin duda que los autores que han sostenido esta posición pecan de ingenuidad epistemológica, cosa común en la literatura científica de origen sajón; desconocen que ya en la observación misma están implicados supuestos de parte del investigador. La teoría no surge nunca de la mera constatación de lo dado, sino de la búsqueda racional de modelos explicativos que den coherencia lógica al material empírico (según ha enfatizado la epistemología de Bachelard).

\subsection{Concepto de fundamentada}

Respecto del concepto de fundamentada (grounded), la credibilidad es un tema importante en la investigación cualitativa: la legitimidad de lo inmediato y de la construcción social realizada. Todo en tal indagación cualitativa se equilibra sobre los significados que el investigador lee en los encuentros sociales que hacemos en la vida.

Para Glaser y Strauss se considera presumido que uno empiece con categorías relevantes e hipótesis. Los escritos posteriores de estos autores intentan desarrollar el concepto de sensibilidad teórica, de algún modo para reconciliar algunas ideas divergentes. Strauss asume una postura más flexible al proponer el uso de una teoría general de la acción para construir un eje de la teoría emergente. Esto es rechazado por Glaser, aunque en sus escritos señala que los códigos teóricos representan los conceptos teóricos de los que dispone el investigador, independientemente de la recolección y análisis de los datos. Los resultados de esta tensión en los enfoques se pueden ver en muchas investigaciones y tesis, se opta por el pragmatismo de la codificación dejando de lado este tipo de cuestiones.

Los referentes teóricos previos no se pueden eliminar y, para el investigador cualitativo, son valiosos y posibles. Debería haber algún supuesto por el cual se elige un determinado campo de estudio. Según Gadamer (1975), el significado existe al comienzo de cualquier investigación y, al terminar le 
permite elegir el tema, desarrolla su deseo de investigar, y permite avanzar en la nueva problemática.

La suspensión de las referencias a las teorías existentes podría permitir un esfuerzo intelectual para descubrir (Guillemette, 2006), pero es ilusorio pensar que se puede abordar un fenómeno sin ningún referente. Los resultados de la investigación no pueden ser puramente construidos a posteriori.

Los límites de la inducción no conducen a adoptar una orientación propiamente deductiva. En la investigación cualitativa, se puede articular la inducción cualitativa con la inferencia abductiva. Esta conjuga el carácter $a$ posteriori de la teorización fundada a partir de los hechos empíricos con el carácter $a$ priori del uso inferencial de la teorías.

Scribano (2001, pág. 4) señala que "en la investigación cualitativa se puede ver claramente como la base empírica de la investigación tiene la forma de una estructura diferenciada en diversos 'niveles' de afirmaciones". Agrega que el investigador incluye en su proceso de análisis lo descriptivo y lo interpretativo, lo que conceptualiza como proceso de abstracción. El investigador trabaja con descripción de los textos y citas textuales con el objetivo de especificar qué significan determinados enunciados. Indudablemente, que hay exigencias de análisis empírico, pero esto se realiza en contexto de una determinada problematización y justificación teórica. Es por ello que Ricoeur (1995) nos alerta al señalar que una cosa es la textura de la acción y otra el texto que escribe el investigador.

En la indagación cualitativa, si la miramos desde la perspectiva de Ricoeur (1970), significa que el investigador expande su "sí mismo" sobre el "sí mismo" del otro; intenta penetrar en un mundo de vivencias y experiencias para conocerlas, describirlas e interpretarlas. Es innegable que este proceso va más allá de lo que significaría la aplicación de un método. En este proceso, se trata de identificar y autoidentificarse; de comprender y comprenderse; de singularizar y ver las diferencias. Y, como señalan los investigadores fenomenológicos, este proceso permite ir generando los énfasis temáticos, los recortes de la realidad, la identificación de textos, intertextos, y contextos. Se encuentra una realidad en la que los actores sociales tienen sus propias interpretaciones, con creencias desde sus particulares perspectivas y el proceso de interpretación se configura a través de redes categoriales de las narrativas, es el paso de lo concreto a lo abstracto. Bien indica Scribano (Ibíd., pág. 5), el investigador trabaja con la fenomenología de la vida cotidiana, y este proceso se entiende como "reconstrucción racional del mundo de la vida donde se encuentran las raíces de un sujeto en el mundo".

Las categorías de la ciencia, la forma, el orden y los conceptos mediadores no son las categorías bajo las cuales una cultura se comprende, piensa y lee a sí misma. De allí que quienes postulan la vigilancia epistemológica, señalen la necesidad de observar el sí mismo que está presente en texto referido al otro.

El investigador cualitativo es un ser humano que escucha, que interpreta a partir de su experiencia como ser humano. Esto, independientemente que se fracturen los datos, se los limpie o se los mapee, uno no estará cerca de la comprensión trascendente. Es decir, no existiría lo fundamentado (grounded) cuando se desarrollan las interpretaciones. La argumentación de la teoría fundamentada pone en evidencia que está implicada en todo aquello que dice trascender (Fish, 1989). 
Glaser y Strauss (1967) diferenciaron la teoría sustantiva de la teoría formal asociando la teoría sustantiva con la investigación concreta de la teoría formal o gran teoría que nace de la comparación entre varias teorías substantivas, que para esta perspectiva, puede llegar a ser universal (Glaser, 2002). La teoría sustantiva tiene relación con la generación de teoría a través de la investigación empírica, mientras que la teoría formal se asocia con el trabajo teórico o conceptual.

Estos autores ven las teorías sustantivas como emergentes mediante la saturación en el análisis de los datos apropiados, la dirección, la cantidad de la recolección de estos datos. Es dirigida por los patrones salientes en dichos datos, más que por un diseño predeterminado de la investigación, lo cual permite generar teorías originales respecto de la conducta humana. El fin último de la teoría fundamentada es generar teorías formales: teorías que pueden ser generalizables en un nivel abstracto. Una teoría formal, desde esta perspectiva, solamente puede emerger a partir de un análisis suficiente de los datos, en casos suficientes, asegurando así que no solamente está describiendo un caso en una situación singular.

Una sola investigación basada en la teoría fundamentada no podría generar una teoría formal. Ésta surge a través del tiempo (Glaser, 1978); y de la reflexión. Así mismo, se deriva de la abstracción conceptual una teoría sustantiva a través de estudios investigativos múltiples (Strauss y Corbin 1998).

Los autores usan la palabra 'grounded' (fundamentada) para recordarnos que el investigador no puede partir investigando con conceptos preconcebidos, un diseño completo o un marco teórico guía (Strauss and Corbin). Uno se puede preguntar cómo los investigadores que asumen esta perspectiva logran colocar en cuarentena su "sí mismo social" respecto a los datos que analizan y reanalizan para permitir que emerja la teoría y cómo pueden permanecer en un territorio neutral. Por otra parte, cuando ellos señalan (Strauss y Corbin 1998, p. 99) que nunca podemos liberarnos completamente de nuestros prejuicios y plantear la necesidad de alejarnos de ellos, no se sabe cómo se podría lograr esta distancia ni el por qué necesitamos distanciarnos. ¿Cómo puede un investigador emerger con algo que no es solamente un informe si como teórico no usa su propia persona para emerger con la teoría? Mucha de la credibilidad de la indagación cualitativa se relaciona con la legitimidad de lo inmediato, y de la construcción social que se realiza.

Supongamos que un investigador que asume la teoría fundamentada registra que la madre le da una bofetada a su hijo porque según ella se portó mal. Se le pregunta a este investigador ¿reaccionó la madre de manera razonable? Difícilmente podría su "sí mismo" olvidar su propia historia, sus propios entre-paréntesis hermenéuticos, todo su conocimiento, sesgos y prejuicios sobre la conducta humana y negar que se hace una interpretación y se responde la pregunta de una u otra manera.

En este punto, el análisis de Scribano (2001, pág. 9), aparece como una aproximación más rigurosa desde el punto de vista epistemológico y metodológico, en especial la relación entre el investigador y el investigado, ambos entendidos como sujetos portadores de interpretaciones. Asimismo, este autor introduce, de algún modo, un proceso de vigilancia epistemológica y metodológica al señalar que es posible corregir las interpre- 
taciones, no así la realidad, incluyendo un análisis crítico de las condiciones de análisis usadas, las precondiciones de visibilidad del objeto de análisis y lo que ha sido la construcción del documento de este análisis. Esto significa, por ejemplo, revisar la relación entre texto, práctica y sentido.

El significado es construido por el intérprete. La relación entre interpretación e intérprete es indisoluble. No hay una verdad oculta de algún modo lista en los datos. No se podría argumentar que el significado sea algo simple, transparente y que recurriendo a él redundaría invariablemente en una explicación satisfactoria. El significado nos remite: por el lado del sentido y la connotación, a toda la subjetividad, tanto a la del investigador como a la de los actores estudiados; y, por el lado de la referencia y la denotación, a todo lo objetivo o a todo lo real (Reynoso, 2007, pág. 23).

Indudablemente, se requiere un proceso de verificación. Esto lo ilustra bien la discusión de Alvesson y Sköldberg (2000) sobre la teoría fundamentada. Estos autores examinaron los textos de Strauss sobre notas de campo de observaciones a enfermeras trabajando en una unidad de recuperación cardíaca donde se ilustra el proceso de codificación. Ellos muestran los significados implícitos en los que se involucra el investigador. Por ejemplo, se alude a la división del trabajo ignorando completamente las dimensiones de género involucradas en la situación. Además, la descripción realizada es altamente técnica y se eliminan las emociones. Consideran que esta forma de proceder neutraliza y reduce la complejidad de una situación a algunas relaciones causales externas que son relativas.

\subsection{Concepto de descubrimiento}

Un tercer concepto que resulta cuestionable es el de "descubrimiento". Este concepto está en el corazón de la teoría fundamentada. Este concepto habla bastante del proyecto epistémico de Glaser y Strauss. Se trata de un proceso de revelar lo que está allí. Y esto se puede lograr siguiendo la aplicación de algún método de hallazgo.

La teoría del descubrimiento significa anclar en los sucesos empíricos y, conceptualizar comunalidades entre ellos, organizados por una categoría nuclear, o comunalidad suprema. Glaser y Strauss reconocen que la producción de teoría es siempre relativa a quien la produce y no hacen ningún intento para justificar esta posición epistemológicamente, ya sea al inicio (Glaser y Strauss, 1967) o en sus amplificaciones y revisiones del método (Glaser) (Strauss y Corbin). Este olvido ha dejado abierta la puerta a posturas epistemológicas alternativas (Madill, Jordan y Shirley, 2000). Glaser y Strauss no logran consenso sobre cómo conducir el método en propiedad (Glaser, 1992; Strauss y Corbin, 1998). Se puede decir que la versión revisada de Strauss es más popular que la versión original defendida por Glaser. A partir de esta bifurcación de la interpretación del método muchos usuarios lo reducen a un mero análisis de temas. Algunos autores persisten en el consenso grupal sobre las categorías. Hay que insistir que lo que producen los investigadores de la teoría fundamentada son interpretaciones, es decir, recurren a la Hermenéutica, aunque rara vez se argumenta en esta dirección.

La idea de que la teoría puede ser descubierta se aleja bastante de la interpretación. El uso de la palabra descubrimiento puede sugerir expectativas cercanas a lo normativo y lo definitivo. 
Se podía decir que, en Glaser y Strauss, descubrir significa acceder a lo que se oculta, a lo que ya existe. Pero esto no es fácil. La revelación de lo que está allí es posible, es decir, se puede acceder a una interpretación trascendente y para ello se requiere aplicar el método de la teoría fundamentada. Mediante este proceso de indagación se puede revelar esta entidad que se oculta.

A diferencia de Glaser y Strauss, en Gadamer la interpretación no es sospecha, es una posibilidad basada en la extrañeza y por tanto la interpretación es la superación de la extrañeza.

Para el investigador cualitativo el sentido no viene dado, hay que construirlo. $Y$ esta tarea es permanente, inacabable y no se entiende el discurso como una colección de enunciados más o menos estructurados: contiene lo dicho y lo no dicho que lo determina. Es un proceso en permanente construcción y reconstrucción. No existe la significación definitiva pero a la vez el discurso aspira a ella de tal manera que, en cada ocasión, en el análisis de cada discurso particular, debiéramos poder encontrar la huella, a la vez, de tal aspiración y de lo que falla en ella. Pero al mismo tiempo se puede observar el "sí mismo que está presente en el texto referido al otro. Al hablar sobre otros se habla desde el "sí mismo. El investigador debe resolver una doble situación: evitar no hacer referencia a los otros y por otra parte ceder a los otros las palabras oscureciendo su presencia.

Por otra parte, el descubrimiento como finalidad normativa, de generalización, olvida que, para la indagación cualitativa, el fenómeno investigado siempre se está resemantizando y reescribiéndose, por lo demás, una de las propuestas de Glaser y Strauss. Se trabaja sobre la mismidad y la diferencia; la identificación de las alteridades y, por otro lado, la semejanza cultural.

$\mathrm{Si}$ bien es cierto que las propias certezas acumuladas bloquean el dar cuenta de lo no vivenciado en su manifestación plural, dado que actuamos analógicamente, desde las semejanzas establecemos identidades a problemas o situaciones que terminan reduciendo y no nos permiten ubicarnos frente al mundo. Esto se aplica a lo teórico y a lo metodológico, que tampoco pueden operar como certeza. Como hacen Glaser y Strauss, las posibilidades del conocimiento no se pueden limitar a seguir un determinado procedimiento. No se puede vivir solamente desde el margen interior sin evitar el esencialismo. En la observación misma están implicados supuestos de parte del investigador, entonces el investigador requeriría de una doble actitud: tomar en serio las palabras de la gente y distanciarse de ellas. Es parte del proceso de lo que se ha denominado vigilancia epistemológica que apunta a develar el por qué y el cómo se piensa, tanto desde la perspectiva del actor social como del investigador. Se requiere comprender el enlace entre la constitución del "sí mismo" en el diálogo con otro "sí mismo", usando un lenguaje ricoeuriano.

La teoría no surge nunca de la mera constatación de lo dado, sino de una interpretación que brinde coherencia lógica al material empírico. Hay que recordar, como argumenta Follari, la ciencia no dice lo real, sino que lo explica por medio de teorías. Ello implica que la ciencia no surge de la observación, como es la creencia más común, sino que implica siempre la existencia de supuestos previos que son contrastados por vía de la experiencia. Este es uno de los puntos que más contradicen la supuesta evidencia: como 
Se mezcla inspiración con predicción y no le es suficiente la comprensión delos fenómenos. Se intentagenerar teorías de alcance medio, más allá de la gran teoría. lo real no habla, sólo se hace inteligible en orden a los interrogantes conceptuales que le son formulados.

Sentido y significación no coinciden. El investigador cualitativo se entera de que dos expresiones diferentes pueden tener la misma referencia, pero distinto sentido. Se puede sustituir, entonces, un signo por otro sin que se modifique la referencia pero esta sustitución no deja inalterado el sentido. De allí que exista una determinada posición de la interpretación respecto del sentido.

De ningún modo, se trata de negar que la formalización lógica encarada como medio para poner a prueba la lógica en el acto de investigación y la coherencia de sus resultados ya que constituyen uno de los instrumentos más eficaces del control epistemológico.

El análisis de Kelle (2005) resulta bastante interesante, aunque significaría alejarse de la teoría fundamentada, la reformulación propuesta simplemente significa asumir una perspectiva epistemológica y metodológica totalmente diferente. Este autor examina la idea de un proceso investigativo basado en lo hipotético o en la inferencia abductiva. El proceso de construcción teórico empezaría por la elección cuidadosa de una variedad de conceptos con antecedentes teóricos divergentes, después examinar su propiedad para la investigación de un determinado fenómeno. Este encuadre heurístico como el eje del desarrollo de la teoría daría paso a la construcción de categorías y proposiciones con creciente contenido empírico. Así mismo, se requeriría la búsqueda de instancias negativas y fenómenos empíricos a los cuales no se aplicarían las categorías heurísticas, lo que significaría reformulación o abandono. Se constituiría entonces la puesta en marcha de un proceso de investigación basado en la inferencia abductiva y con- sistentemente falible. Dicho de otra manera, no se argumenta que la validez de las proposiciones desarrolladas a partir de los datos empíricos. Se podría establecer simplemente por el hecho de que el investigador haya liberado su mente de cualquier pre-concepción antes de recopilar los datos. Se enfatiza en las elaboraciones teóricas más allá de las meras descripciones de los hechos observados, pero deben considerarse como falibles.

\section{Los procesos de codificación y categorización}

La codificación de las unidades textuales que recopila el investigador cualitativo tiene como propósito reducir, categorizar o construir el significado presente en el material. La teoría fundamentada emplea varias etapas, abierta, axial, y selectiva, en orden a construir teoría sobre el fenómeno estudiado. En el análisis de los textos, la codificación es una etapa necesaria en orden a explorar el significado. Dos tópicos comunes son cómo la codificación puede reducir el material empírico y cómo se pueden crear, ordenar y amalgamar teóricamente los códigos. La comprensión del significado es fundamental como trasfondo de los procesos de codificación.

El método de la teoría fundamentada hace un desarrollo bastante específico de lo que significa la codificación y la categorización de los textos obtenidos por la investigación cualitativa. No se puede desconocer que el procedimiento propuesto es bastante útil, de allí su popularidad. No obstante, lo que resulta limitado es la aproximación de la teoría al proceso de análisis e interpretación de la categorización. Es por ello que se desarrolla en los párrafos una aproximación que pretende precisar que al codificar y categorizar no se trata de una cuestión inductiva de formalización conceptual. 
El investigador puede interesarse en el significado referido a las intenciones, propósitos, motivos, entre otros, explícitos del propio autor. Este es el lado subjetivo del significado. O el interés puede ser lo que dice. Ya no es una cuestión sobre el significado intentado por el autor, concierne con el aspecto comprensivo y comunicativo del lenguaje. El análisis puede estar enfocado al concepto de referencia antecedente, consistente en explorar sobre lo que habla el texto, sobre lo que existe, independientemente de la intención del autor. Finalmente, el foco podría ser el significado relacionado a posibilidades futuras presentes en el texto, es decir, aquello que puede llegar a ser: proposiciones o posibilidades. Es el concepto de referencia hacia adelante.

Los textos, entrevistas en profundidad, entrevistas grupales, y demás referencias son desglosados, reestructurados, y analizados por el investigador dando como resultado explicaciones en términos de códigos con- ceptuales. Por ejemplo, respuestas a preguntas sobre qué, cómo, y quién. Esta explicación además tiene un elemento cognitivo ya que tanto el investigador como la persona que lee, requiere que la explicación sea entendible.

El investigador identifica las unidades de significado, las cuales pueden variar en tamaño desde una palabra a párrafos completos. Los elementos textuales aislados en el procedimiento de codificación son agrupados con otros elementos en el proceso de análisis, segmentación que genera relaciones horizontales entre los elementos. Las unidades condensadas de significado se crean a través de la detección de similitudes y diferencias. Finalmente, las unidades de significado se abstraen en subtemas, temas y temas principales. La Tabla 1 muestra una parte del proceso de análisis referido a una madre y su hijo drogadicto. La Tabla 2 permite observar el análisis temático.

\section{Tabla 1.Ejemplo de análisis}

\begin{tabular}{|c|c|c|c|}
\hline Unidad de significado & Unidad de significado condensado & Sub-tema & Tema \\
\hline $\begin{array}{l}\text { Segmento textual: } \\
\text { "Podemos sobrellevar la situación } \\
\text { aunque es difícil. Pero no sabemos } \\
\text { cómo reaccionará él... uno nunca } \\
\text { sabe si ocurrirá algo... entonces uno } \\
\text { no sabe cómo actuará". }\end{array}$ & $\begin{array}{l}\text { Como van las cosas, se están adaptando } \\
\text { a la situación. La madre muestra } \\
\text { preocupación respecto al tratamiento } \\
\text { que está recibiendo su hijo. Tiene } \\
\text { preocupación por posibles efectos del } \\
\text { tratamiento. }\end{array}$ & $\begin{array}{l}\text { Vivir con } \\
\text { una tensión } \\
\text { permanente }\end{array}$ & $\begin{array}{l}\text { La lucha } \\
\text { de cada } \\
\text { día }\end{array}$ \\
\hline
\end{tabular}

\section{Tabla 2. Temas y subtemas}

\begin{tabular}{|l|l|}
\hline Temas & Sub-temas \\
\hline \multirow{3}{*}{ Sentimiento de limitación } & Existencia limitada \\
& Disminución de la espontaneidad \\
& Disminución de las relaciones sociales \\
& Dificultad para imponer límites \\
& Adaptación permanente \\
\hline \multirow{4}{*}{ Lucha de cada día } & Cambios en las relaciones con otros \\
& Vivir el día a día \\
& Preservar el estilo de vida previo \\
Luchas por la normalización & Tratar de recuperar el equilibrio emocional \\
& Apoyo de otros \\
& Colocarse uno mismo en el centro de atención
\end{tabular}


Se requiere también que el análisis aborde qué es el texto. ¿Qué información provee sobre el mundo? ¿Qué dimensiones de ser en el mundo se abren en el texto? ¿Cuál es la verdad que sustenta? Esto se logra como resultado del distanciamiento que significa una retirada de las referencias directas y literales. El sentido del texto no es algo oculto sino algo que se revela, cuya comprensión significa seguir los movimientos desde el sentido a la referencia; desde lo que se dice a lo qué se habla.

Un proceso de acercamiento y distancia permite profundizar nuestra compresión de las experiencias vividas por lo sujetos. Para obtener distancia del texto, se lo lee y examina gramaticalmente; se estudian los pronombres, tiempos verbales y negaciones; se estudian determinados tipos de frases como causalidad, probabilidad, etc. Se exploran patrones en el texto, posibles cambios dramáticos y momentos decisivos o cruciales. El investigador puede analizar las diferentes voces presentes en el texto, las descripciones metafóricas que pudieren representar tramas como ¿qué clase de historias se cuentan? ¿Qué explicaciones ofrecen los sujetos de sus propias historias? ¿Cómo relatan su pasado respecto de su condición actual?

El investigador enfrenta el problema de transformar varias narrativas individuales en una narrativa colectiva durante el proceso de codificación. ¿Se deben tratar los códigos como singularidades únicas o como agregados en códigos más genéricos? ¿Cómo se pueden conectar los códigos, simples o agregados, en las interpretaciones globales?

Habría distintos modelos para la codificación de los datos. En primer término el investigador construye una matriz de codificación. Es importante que el investigador defina los sujetos que quiere comparar y/o contrastar, de modo que el análisis temático permita explorar similitudes y/o diferencias entre los sujetos que forman parte del análisis.

\section{Tabla 3. Matriz de codificación}

\begin{tabular}{|l|l|l|l|l|}
\hline Lectura & \multicolumn{3}{|c|}{ Análisis estructural } & Comprensión \\
\hline $\begin{array}{l}\text { Unidades } \\
\text { preliminares de } \\
\text { significados }\end{array}$ & Qué códigos & $\begin{array}{l}\text { Quién } \\
\text { Dónde }\end{array}$ & $\begin{array}{l}\text { Códigos } \\
\text { reflexivos }\end{array}$ & \\
\hline
\end{tabular}

Un modelo significaría: leer el texto obteniendo varias comprensiones como un todo según los textos existan. Se analiza cada historia estructuralmente lo que genera un equis número de códigos para cada historia. El paso siguiente, la comprensión y los códigos de cada historia se unen en una comprensión de cada historia. Luego, la comprensión de todas las historias se unen en una comprensión agre- gada del material como un todo, y esto podría formar la base para la apropiación eventual. Esta forma de análisis de los textos puede ser adecuada si el material es muy heterogéneo, es decir, si las historias difieren unas de otras a pesar del tema principal.

Un segundo modelo implicaría, como primera medida, leer el texto y proceder a compilar memorandos para cada comprensión 
del material como un todo. El paso siguiente sería un análisis estructural de cada historia. Los códigos obtenidos por el análisis estructural de todas las historias se juntan en una totalidad. Seguidamente, la totalidad de los códigos y la comprensión obtenida se unen en una comprensión de todas las historias, lo cual sienta las bases para la apropiación. Este modo de análisis es adecuado si se cuenta con material empírico relativamente homogéneo.

Los textos pueden desde algún punto de vista resultar únicos, pero se puede encontrar un denominador común como lo son tipos de adictos, tipos de intervenciones, tipos de conocimiento, entre otros, que haría posible compilar una comprensión del material como un todo. No obstante, los textos podrían contener numerosos componentes únicos entre los que se encuentran descripciones y sentimientos, que solamente permitirían analizar estructuralmente las historias individualmente.

La preocupación no es identificar cada caso bajo una categoría general; el conocimiento procede por analogía, donde un individuo puede ser o no ser similar a otros. Lo que importa son los sentidos singulares que expresan y las lógicas particulares de argumentación que despliegan. El significado no puede ser creado intuitivamente, este emerge en el proceso interpretativo que incluye explicación y comprensión.

Complementando lo expuesto en los párrafos previos, el estudio de los textos reunidos por el investigador cualitativo puede ser empleado como unidades de análisis: palabra, proposición, frase, párrafo, texto(s). El investigador puede considerar las palabras en su contexto mediante un análisis lexicográfico. En su estudio puede identificar que determinado conjunto de palabras que aparecen en el texto pueden evocar cuestiones temáticas identificadas en los análisis teóricos que son antecedentes de la obtención de una narrativa, por ejemplo, ciertas palabras pueden evocar distintos tipos de cultura por medio de un diálogo, comunicación, consenso, personas, grupos.

Esto significa que el investigador establece las unidades mínimas de registro y de análisis, que son gramaticales, pragmáticas y de sentido social, así como las interacciones situacionales identificables en la narrativa; explora el significado denotado a cada unidad mínima o proposición y contextualizarla en sus propios escenarios; analiza la variedad de relaciones verbales por medio de categorías, así como las relaciones verbales empíricas, procesos fácticos, procesos cognitivos presentes en las narrativas; puede estudiar las estrategias discursivas; e identifica los temas referenciales, actores y otros contenidos de la interacción, procediendo a reducirlos y codificarlos.

El estudio del texto permite identificar: el sujeto de la acción o quién, los eventos o actividades refiriéndose al qué, la intención o el para qué, las razones de la actuación o por qué, el proceso cómo y a los deícticos dónde y cuándo.

En este marco, la interpretación parte de un elemento objetivo formal o mensaje, las referencias, “¿Acerca de qué?” Otro elemento subjetivo, "el contenido”, el significado, “qué?”, la dimensión autorreferencial (conocimiento previo), elocutivo (hacer algo) e intencional (¿para qué?).

El investigador realiza un proceso de análisis pasando de una fase de interpretación a otra analítica y profunda ubicando la explicación y comprensión en dos distintos momentos. En este proceso los conceptos interpretados no son temáticos como tales, al 
El significado es construido por

el intérprete. La relación entre interpretación e intérprete es indisoluble. No hay una verdad oculta de algún modo lista en los datos. No se podría argumentar que el significado sea algo simple, transparente $y$ que recurriendo a él redundaría contrario, son herramientas que se usan para el proceso de teorización. La interpretación que se hace no se queda en las temáticas, son puentes que ayudan a representar un determinado proceso teórico sobre lo que se está estudiando. Este proceso permitirá identificar elementos cercanos, vecinos, consecutivos o contrapuestos según sea el caso.

\section{A modo de conclusión}

Un argumento sobre el éxito de la teoría fundamentada, se podría decir, que deriva de que ha ayudado a validar la indagación cualitativa. No obstante, las argumentaciones anteriores permitirían señalar que hay demasiadas cuestiones no resueltas por esta teoría haciendo innecesaria su reformulación. Lo más importante es tener claridad de los vacíos y ambigüedades de esta teoría que afecta la indagación cualitativa. La teoría fundamentada privilegia determinada forma de pensamiento, impone cierta racionalidad y formas de proceder, relegando la voz original, o narrativa, de los participantes. La insinuación de que hay que interpretar, mediante un determinado método la alteridad, la narrativa del otro de modo que sea entendida racionalmente mediante la fractura de los datos, de modo que develar el sentido, compromete seriamente el sentido de la indagación cualitativa. El énfasis en los procedimientos desplaza el valor investigativo del fin a los medios. La indagación cualitativa no es solamente un campo de análisis de saturaciones, codificaciones y muestra teóricas.

El investigador busca capturar los aspectos discursivos de orden macrotextual y no necesariamente segmentos aislados o códigos aislados. Las relaciones que se mapean no son simplemente de ocurrencia derivada sino que obedecen a un amplio rango de funciones semánticas como la causalidad, pertenencia, identidad, inclusión tópica, sucesión temporal, afinidad cognitiva, entre otras. La elección de los verbos no es accidental: podríamos aludir a toda una teoría de la variación para dar cuenta del carácter intencional de las elecciones léxicas. La adjetivación de agentes, objetos y acciones, además del uso de verbos con carga axiológica intrínseca, son los recursos de indudable incidencia valorativa. Las descripciones factuales capaces de darle un contenido climático a la narrativa del actor social. Sus procesos de análisis deberían permitir vincular los niveles micro con los macro sin olvidar en ningún momento que las herramientas no son teorías, aunque puedan estar al servicio de ellas. No importa cuán seductor y fructífero parezca un instrumento, una técnica está muy bien en el lugar que le cabe pero no satisface el papel de una teoría, como aprendimos por la vía cruel en nuestra disciplina cuando se intentó la aventura del análisis componencial, nos comenta Reynoso (2008).

Finalmente, el camino para atender con seriedad al actor social es preocuparse, tanto de los procesos cognitivo-simbólicos como sociales que contribuyen a que articule su realidad y que actúe tal como lo hace. El investigador no puede renunciar a comparar e identificar procesos que sirvan de base para generalizaciones parciales a partir de las narrativas. La repetición y diversificación de los casos permite atender a las variaciones, a los parecidos y a las diferencias tanto prácticas como simbólicas a las que las narrativas apuntan individualmente, de este modo, se pueden identificar espacios históricos, sociales, y cognitivos plausibles en los cuales se puedan asentar las hipótesis investigativas.

Es por ello, que los investigadores que asumen la teoría fundamentada deberían 
hacer un esfuerzo teórico más allá del pragmatismo de sus decisiones, en especial porque su uso no clarifica la pre comprensión, dificulta el uso del conocimiento teórico explícito, eleva una forma de pensar desconociendo otros procedimientos de comprensión e interpretación, relega la voz original de la narrativa del que responde y ante las interrogantes sobre una situación, un delito, una adicción, un mal rendimiento u otro factor, desconoce el papel del que interpreta con sus sesgos y sus pre-conceptos, entre otros. En esto, habría que recodar lo que Lyotard denomina como el habla privilegiada de la comunidad (Haber, 1994). Por otra parte, se relega la necesaria cuenta de los investigadores mismos y por ende se carece de vigilancia epistemológica.

Hay cuestiones que parecen insuperables, salvo cambiando la esencia de la propuesta de la teoría fundamentada, en lo concerniente a epistemología y metodología, específicamente a los análisis sobre la generación y desarrollo de teorías. Desde nuestro punto de vista, así como no es sostenible la no neta derivación deductiva de la teoría sin atender especificidades, tampoco sería acertado asumir un enfoque en el que no hay posibilidad de contar con criterios teóricos desde los cuales situarse, porque no se trata de sostener que la teoría es un aporte rechazable y que se trata simplemente de hacer hablar a los actores.

El enfoque de la investigación cualitativa desde la perspectiva de la abducción puede resultar no solamente relevante, sino que significativo al momento de realizar los análisis con los datos obtenidos empíricamente. Mediante la abducción se pueden conectar modelos preexistentes con configuraciones de hechos y, a través de este proceso, se logra una interacción de las teorías de las que dispone el investigador y la experiencia de los participantes en la investigación, lo que supone una re significación de ambos, como se ha aludido previamente (Samaja, 2007). Además, si se integra el concepto de vigilancia epistemológica como componente sustantivo del proceso investigativo que signifique que el investigador dé cuenta de sus elecciones, de la teoría desde la que hace la interpretación clarificando así los conceptos usados, las proposiciones e hipótesis, se estaría en una senda interesante de indagación cualitativa. Pero un enfoque de esta índole es totalmente diferente a la teoría fundamentada.

Un tema a resolver, que escapa a este artículo, es si la forma de proceder de la teoría fundamentada es o no inconmensurable con un enfoque como la abducción o la fenomenología hermenéutica.

\section{Bibliografía}

1. Altricher, H., \& Posch, P. (1989). Does the "grounded theory" approach offer a guiding paradigm for teacher research? Cambridge Journal of Education, 19(1), 21-31.

2. Alvesson, M. \& Sköldberg, K. (2000) Reflexive Methodology - New Vistas For Oualitative Research. London: Sage.

3. Annells M. (1997a). Grounded theory method, Part I: within the five moments of qualitative research. Nursing Inquiry, 4(2), 120-129.

4. Annells M. (1997b) Grounded theory method, Part II: options for users of the method. Nursing Inquiry, 4(3), 176-180. 
5. Annells M. (2006).Triangulation of qualitative approaches: hermeneutical phenomenology and grounded theory. Journal of Advanced Nursing 56(1), 55-61

6. Ashworth, P. D. (1996). Presuppose nothing! The suspension of assumptions in phenomenological psychological methodology. Journal of Phenomenological Psychology, (27), 1-25.

7. Charmaz, K. (1995) Grounded theory. In J.A. Smith, R. Harre and L. Van Langenhove (eds.) Rethinking Method in Psychology (pp. 27-49). London: Sage.

8. Charmaz, K. (2000) Grounded theory: Objectivist and constructivist methods. In N.K. Denzin and Y.E.Lincoln (eds) Handbook of qualitative research (2nd ed) (pp. 509-535). Thousand Oaks, CA: Sage.

9. Clarke. A. 2005. Situational Analysis: Grounded Theory After the Postmodern Turn. Thousand Oaks, CA: Sage.

10. Dey, I. (1999) Grounding Grounded Theory -GuidelinesforQualitative Inquiry London: Academic Press.

11. Fish, S. (1985) Consequences. In W.J.T. Mitchell (Ed.), Against Theory: Literary Studies and the New Pragmatism (pp. 106-131) Chicago: University of Chicago Press.

12. Fish, S. (1989) Doing what Comes Naturally. Oxford: Clarendon Press.

13. Fish, S. (1994) There is no such thing as free speech. Oxford: Oxford University Press.

14. Gadamer, H-G. (1975) Truth and Method. London: Sheed \& Ward.

15. Glaser,B.G. \& Strauss, A.L. (1967) The Discovery ofGrounded Theory: strategies for Qualitative Researc: $h$. New York:Aldine.

16. Glaser, B.G. (1978) Theoretical sensitivity. Mill Valley, CA: Sociology Press.

17. Glaser, B.G. (1992) Basics of Grounded Theory Analysis. Mill Valley, CA: Sociology Press.

18. González Echeverría, A. (2006). Del utillaje conceptual de la antropología: los usos del término 'inductismo' y los usos del término 'herméutica'. Dos propuestas de clarificación. Revista de Antropología Social, 15, pág. 327-372.

19. Guillemette, F. (2006). L'approche de la Grounded Theory, pour innover? Recherches qualitatives, 26(1), 32-50.

20. Haber, F.H. (1994) Beyond Postmodern Politics: Lyotard, Rorty, Foucault. London: Routledge.

21. Hammerley, M. (1985) From ethnography to theory: a programme and paradigm in the sociology ofeducation, Sociology, 19(2) 244-259

22. Hammerley, M (1992) What's Wrong with Ethnography? London: Routledge.

23. Layder, D. (1993) New Strategies in Social Research: an Introduction and Guide. Cambridge: Polity Press.

24. Layder, D. (1998) Sociological Practice: Linking Theory and Social Research. London: Sage.

25. Lincoln, Y.S. \& Guba E.G. (1985) Naturalistic Inquiry. Beverly Hills: Sage.

26. Madill, A., Jordan, A. y Shirley, C. (2000). Objectivity and reliability in qualitative analysis: Realist, contextualist and radical constructionist epistemologies. British Journal of Psychology, 91, 1-20. 
27. Miller, S. \& Fredericks M. (1999) How does grounded theory explain? Qualitative Inquiry, 9, 538-551.

28. Mouzelis, N. (1995) Sociological Theory: What Went Wrong? London: Routledge.

29. Nadel, S. F. (1957) The Theory of Social Structure. London: Cohen \& West.

30. Ricoeur, P. (1970) Freud and Philosophy: An Essay on Interpretation. New Haven: Yale University Press.

31. Ricoeur, P. (1995). Tiempo y narración I: La configuración del tiempo en el relato histórico. México: Siglo XXI.

32. Samaja, J. (2007). Epistemología y Metodología. Buenos Aires: Eudeba

33. Scribano, A. (2001). Investigación cualitativa y textualidad. Cinta de Moebio, Revista Electrónica de Ciencias Sociales, 11, pág. 1-12.

34. Strauss, A. \& Corbin, J. (Eds.) (1997) Grounded Tbeory in Practice. Thousand Oaks, CA: Sage.

35. Strauss, A. \& Corbin, J. (1998) Basics of Qualitative Research. Techiques and Proceduresfor Deve! oping GroundedTbeory. Thousand Oaks, CA: Sage.

36. Strauss, A. (1987) Qualitative Analysisfor Social Scientists. Cambridge: Cambridge University Press.

37. Thomas, G. (1997) What's the use of theory? Harvard Educational Review, 67, pp. 75-105.

38. Wacquant, L.D. (1989) Towards a reflexive sociology: a workshop with Pierre Bourdieu. SociologicalTheory, 7,26-63.

\section{Consultas en línea}

1. Follari, R (s/f). La ciencia como real maravilloso. Recuperado de http://www. redcientifica.com/doc/doc200111120001.html

2. Haig, Brian D. (1996) Grounded theory as scientific method. Recuperado desde: http://www.ed.uiuc.edu/EPS/PES-yearbook/95_docs/haig.html

3. Kelle, U. (2005). "Emergence" vs. "Forcing" of Empirical Data? A Crucial Problem of "Grounded Theory "Reconsidered. Forum Qualitative Research. 6 (2). Art. 27. Recuperado de http://www.qualitative-research.net/index.php/fqs/article/ viewArticle/467/1000

4. Reynoso, C. (2007). El lado oscuro de la descripción densa. Recuperado de: http:// carlosreynoso.com.ar/el-lado-oscuro-de-la-descripcion-densa-version-2007/ 